\title{
A LÉVY INSURANCE RISK PROCESS WITH TAX
}

\author{
HANSJÖRG ALBRECHER, ${ }^{* *}$ Austrian Academy of Sciences and University of Linz \\ JEAN-FRANÇOIS RENAUD, ${ }^{* * *}$ Austrian Academy of Sciences \\ XIAOWEN ZHOU, ${ }^{* * * *}$ Concordia University
}

\begin{abstract}
Using fluctuation theory, we solve the two-sided exit problem and identify the ruin probability for a general spectrally negative Lévy risk process with tax payments of a loss-carry-forward type. We study arbitrary moments of the discounted total amount of tax payments and determine the surplus level to start taxation which maximises the expected discounted aggregate income for the tax authority in this model. The results considerably generalise those for the Cramér-Lundberg risk model with tax.
\end{abstract}

Keywords: Lévy process; fluctuation theory; excursion theory; scale functions; insurance risk theory; ruin probability; tax payments

2000 Mathematics Subject Classification: Primary 60G51; 91B30

Secondary $60 \mathrm{~J} 75$

\section{Introduction}

The classical risk model describes the surplus process of an insurance company by a stochastic process $U=(U(t))_{t \geq 0}$ with

$$
U(t)=u+c t-S(t)
$$

where $S(t)$ is a compound Poisson process with jump intensity $\theta$ and jump distribution $F$ (representing the aggregate claim payments up to time $t$ ), $u>0$ denotes the initial surplus, and $c>0$ is a constant premium intensity. Usually it is assumed that the net profit condition

$$
c>\theta \mu
$$

holds, where $\mu$ denotes the expected value of the single claim size distribution $F$. This condition ensures that ruin will not occur almost surely. As a Lévy process, $U$ has a characteristic exponent given by

$$
\Psi(\lambda)=-\ln \mathrm{E}\left[\mathrm{e}^{\mathrm{i} \lambda(U(1)-u)}\right]=-\mathrm{i} \lambda c-\int_{-\infty}^{0}\left(\mathrm{e}^{\mathrm{i} \lambda z}-1\right) \theta F(\mathrm{~d} z)
$$

for $\lambda \in \mathbb{R}$.

Received 7 December 2007; revision received 5 March 2008.

* Postal address: Johann Radon Institute for Computational and Applied Mathematics, Austrian Academy of Sciences, Altenbergerstrasse 69, A-4040 Linz, Austria.

** Email address: hansjoerg.albrecher@oeaw.ac.at Supported by the Austrian Science Fund Project P18392.

*** Email address: jean-francois.renaud@ oeaw.ac.at

**** Postal address: Department of Mathematics and Statistics, Concordia University, 1455 de Maisonneuve Blvd W., Montréal, Québec, H3G 1M8, Canada. Email address: xzhou@mathstat.concordia.ca

Supported by an NSERC grant. 
One way to generalise the classical risk process is to consider an arbitrary spectrally negative Lévy process, i.e. a process $X=(X(t))_{t \geq 0}$ with independent and stationary increments and with characteristic exponent given by

$$
\Psi(\lambda)=-\mathrm{i} \lambda c+\frac{1}{2} \sigma^{2} \lambda^{2}-\int_{-\infty}^{0}\left(\mathrm{e}^{\mathrm{i} \lambda z}-1-\mathrm{i} z \mathbf{1}_{\{z>-1\}}\right) \Pi(\mathrm{d} z)
$$

for $\lambda \in \mathbb{R}$ and $\sigma \geq 0$, where $\Pi$ is a measure on $(-\infty, 0)$ such that

$$
\int_{-\infty}^{0}\left(1 \wedge z^{2}\right) \Pi(\mathrm{d} z)<\infty
$$

Here, $c>0$ again represents the constant premium intensity. The net profit condition for this Lévy insurance risk process now reads

$$
\mathrm{E}[X(1)-u]>0
$$

which is equivalent to $\lim _{t \rightarrow \infty} X(t)=\infty$ almost surely.

An interpretation of such Lévy risk processes for the surplus modelling of large insurance companies is, for instance, given in [12] and [15]. This model has recently attracted a lot of research interest; see, e.g. [6], [8], [9], [11], [13], and [18].

In a recent paper, Albrecher and Hipp [1] investigated how tax payments (according to a loss-carry-forward system) affect the behaviour of a Cramér-Lundberg surplus process. In their model taxes are paid at a fixed proportional rate $\gamma$ whenever the company is in a profitable situation, defined as being at a running maximum of the surplus process. It turned out that in this model there is a strikingly simple relationship between ruin probabilities with and without tax, and that we could also obtain an explicit formula for the expected discounted sum of tax payments over the lifetime of the risk process.

In this paper we will embed this tax model into a general Lévy framework. Utilising excursion theory and exploiting the structure of the model, we will solve the two-sided exit problem for the Lévy risk process with tax and, consequently, recover the simple relation between ruin probabilities with and without tax in this more general class of models. Furthermore, expressions for arbitrary moments of discounted tax payments until ruin will be derived. It turns out that close connections of the distribution of tax payments to the distribution of dividend payments according to a horizontal barrier strategy, that were observed in the Cramér-Lundberg model, carry over to the Lévy setup.

The paper is organised as follows. In Section 2 we will review some preliminaries on spectrally negative Lévy processes that will be needed later on. In Section 3 we introduce the tax model under consideration and then derive a new fluctuation identity, the ruin probability, as well as moments of discounted tax payments until ruin. Finally, in Section 4 the problem of an optimal choice of a threshold surplus level for starting taxation to maximise the expected tax income will be addressed.

\section{Preliminaries on spectrally negative Lévy processes}

Let $X=(X(t))_{t \geq 0}$ be a spectrally negative Lévy process or, in other words, a Lévy process with no positive jumps (to avoid trivialities, we exclude the case where $X$ is a negative subordinator or a deterministic drift). The law of $X$ such that $X(0)=u \geq 0$ will be denoted by $\mathrm{P}_{u}$ and the corresponding expectation by $\mathrm{E}_{u}$ (for a general introduction to Lévy processes, we refer the reader to [3] or [14]). 
As the Lévy process $X$ has no positive jumps, its Laplace transform is given by

$$
\mathrm{E}_{u}\left[\mathrm{e}^{\lambda(X(t)-u)}\right]=\mathrm{e}^{t \psi(\lambda)}
$$

for $\lambda \geq 0$ and $t \geq 0$, where $\psi(\lambda)=-\Psi(-\mathrm{i} \lambda)$. In this case the Laplace exponent $\psi$ is strictly convex and $\lim _{\lambda \rightarrow \infty} \psi(\lambda)=\infty$. Thus, there exists a function $\Phi:[0, \infty) \rightarrow[0, \infty)$ such that

$$
\psi(\Phi(\lambda))=\lambda, \quad \lambda \geq 0
$$

We now define the so-called scale functions $\left\{W^{(q)} ; q \geq 0\right\}$ of the process $X$ as in [4]. For each $q, W^{(q)}:[0, \infty) \rightarrow[0, \infty)$ is the unique, strictly increasing, and continuous function with Laplace transform

$$
\int_{0}^{\infty} \mathrm{e}^{-\lambda z} W^{(q)}(z) \mathrm{d} z=\frac{1}{\psi(\lambda)-q}
$$

for $\lambda>\Phi(q)$. When $q=0$, we write $W$ instead of $W^{(0)}$.

\subsection{Two-sided exit problem}

Scale functions arise naturally when considering two-sided exit problems for spectrally negative Lévy processes. Indeed, let $a$ be a positive real number and define $\tau_{a}^{+}=\inf \{t>$ $0: X(t)>a\}$ and $\tau_{0}^{-}=\inf \{t>0: X(t)<0\}$ with the convention inf $\varnothing=\infty$. When the process $X$ starts within the interval (i.e. $X(0)=u \in(0, a)$ ), the random time $\tau_{a}^{+} \wedge \tau_{0}^{-}$is the first exit time of $X$ from this interval. Since $X$ has no positive jumps, it will hit the point $a$ when exiting above, but it might jump below 0 when exiting below. Its Laplace transform on the event where the process $X$ leaves the interval at the upper boundary is given by

$$
\mathrm{E}_{u}\left[\exp \left(-q \tau_{a}^{+}\right) \mathbf{1}_{\left\{\tau_{a}^{+}<\tau_{0}^{-}\right\}}\right]=\frac{W^{(q)}(u)}{W^{(q)}(a)}, \quad q \geq 0 .
$$

Consequently, when $q=0$,

$$
\mathrm{P}_{u}\left\{\tau_{a}^{+}<\tau_{0}^{-}\right\}=\frac{W(u)}{W(a)} .
$$

If $X$ has a positive mean, we have

$$
\mathrm{P}_{u}\left\{\inf _{t \geq 0} X(t) \geq 0\right\}=\psi^{\prime}(0+) W(u) .
$$

This result is of course related to the ruin and survival probabilities in insurance risk theory.

\subsection{Smoothness of the scale functions}

At several places in this paper, differentiability of the scale functions will be required. If the sample paths of $X$ are of unbounded variation then the scale functions $W^{(q)}$ are continuously differentiable. When the sample paths of $X$ are of bounded variation then the scale functions are continuously differentiable if and only if $\Pi$ has no atoms or, in other words, if $\{x<0 \mid \Pi(\{x\})>0\}=\varnothing$. Note that if $X$ has a Gaussian component then its sample paths are of unbounded variation and, moreover, its scale functions are even twice continuously differentiable. Furthermore, if the Lévy measure $\Pi$ has a density then the scale functions are always differentiable (see [5] or [7] for more details). 


\section{The model}

Let $X$ be the underlying Lévy risk process with differentiable scale functions. Let $S^{X}=$ $\left(S^{X}(t)\right)_{t \geq 0}$ denote the running maximum of $X$, i.e. $S^{X}(t)=\max _{0 \leq s \leq t} X(s)$. This process is continuous and, of course, increasing. Clearly, $S^{X}(0)=u$ as $X(0)=u$. For $0 \leq \gamma \leq 1$, define a process $U_{\gamma}=\left(U_{\gamma}(t)\right)_{t \geq 0}$ by

$$
U_{\gamma}(t)=X(t)-\gamma\left(S^{X}(t)-X(0)\right) .
$$

We can think of $U_{\gamma}$ as the surplus process of an insurance company that pays out taxes at a fixed rate $\gamma$ whenever it is in a profitable situation (or, in other words, whenever the surplus is at a running maximum). When $\gamma=1$, this amounts to the situation where the company pays out as dividends any capital above its initial value.

\subsection{A fluctuation identity}

The following theorem generalises both Theorem VII.8 of [3] and (2.1).

Theorem 3.1. For any $0<u<a$ and $q \geq 0$, let $\tau_{a, \gamma}^{+}=\inf \left\{t>0: U_{\gamma}(t)>a\right\}$ and $\tau_{0, \gamma}^{-}=$ $\inf \left\{t>0: U_{\gamma}(t)<0\right\}$. If $\gamma<1$ then

$$
\mathrm{E}_{u}\left[\exp \left(-q \tau_{a, \gamma}^{+}\right) \mathbf{1}_{\left\{\tau_{a, \gamma}^{+}<\tau_{0, \gamma}^{-}\right\}}\right]=\left(\frac{W^{(q)}(u)}{W^{(q)}(a)}\right)^{1 /(1-\gamma)} .
$$

Proof. We first consider the case that $X$ drifts to positive infinity, and we want to prove (3.1) for $q=0$.

It is well known that $S^{X}$ is a local time at 0 for the Markov process $S^{X}-X$. Then, let $\varepsilon$ be the excursion process of $S^{X}-X$ away from 0 , and let $\bar{\varepsilon}$ be the excursion height process. If $X$ drifts to $\infty$ then $\varepsilon$ is a Poisson point process and $\bar{\varepsilon}$ is also a Poisson point process with characteristic measure $v$ given by $v(x, \infty)=W^{\prime}(x) / W(x)$. By the definition of an excursion, the event $\left\{\tau_{a, \gamma}^{+}<\tau_{0, \gamma}^{-}\right\}$is the same as

$$
\left\{\bar{\varepsilon}_{s}<u+(1-\gamma) s \text { for all } 0 \leq s \leq \frac{a-u}{1-\gamma}\right\} .
$$

Indeed, if an excursion starts from level $u+s$ (for the process $X$ ), meaning that the process $U_{\gamma}$ is at level $u+(1-\gamma) s$, to avoid falling below 0 , this excursion must not exceed the latter quantity; this must be true until $U_{\gamma}$ reaches level $a$, which is equivalent to $X$ reaching level $a /(1-\gamma)$. Then, by the definition of a Poisson point process, we have

$$
\begin{aligned}
\mathrm{P}_{u}\left\{\tau_{a, \gamma}^{+}<\tau_{0, \gamma}^{-}\right\} & =\mathrm{P}\{N=0\} \\
& =\exp \left(-\int_{0}^{(a-u) /(1-\gamma)} \frac{W^{\prime}(u+(1-\gamma) s)}{W(u+(1-\gamma) s)} \mathrm{d} s\right) \\
& =\exp \left(-\frac{1}{1-\gamma} \int_{0}^{a-u} \frac{W^{\prime}(u+s)}{W(u+s)} \mathrm{d} s\right) \\
& =\left(\frac{W(u)}{W(a)}\right)^{1 /(1-\gamma)},
\end{aligned}
$$

where $N$ is a Poisson distributed random variable with parameter

$$
\int_{0}^{(a-u) /(1-\gamma)} v(u+(1-\gamma) s, \infty) \mathrm{d} s
$$


that counts the number of Poisson points $\left(s, \bar{\varepsilon}_{s}\right)$ in

$$
\left\{(x, y) \in \mathbb{R}^{2} \mid 0 \leq x \leq \frac{a-u}{1-\gamma}, u+(1-\gamma) x \leq y\right\} .
$$

Without assuming that $X$ drifts to $+\infty$, for $q>0$, we define another probability measure $\mathrm{P}_{u}^{\Phi(q)}$ on $\left(\mathcal{F}_{t}\right)_{t \geq 0}$ with Radon-Nikodym derivative

$$
\frac{\mathrm{dP}_{u}^{\Phi(q)}}{\mathrm{dP}}=\mathrm{e}^{\Phi(q)(X(t)-u)-q t}
$$

on $\mathcal{F}_{t}$, where $\left(\mathcal{F}_{t}\right)_{t \geq 0}$ denotes the filtration generated by $X$. Under $\mathrm{P}_{u}^{\Phi(q)}, X$ is still a spectrally negative Lévy process, but now with $W_{\Phi(q)}$ as its 0 -scale function, which are given by $\mathrm{e}^{\Phi(q) x} W_{\Phi(q)}(x)=W^{(q)}(x)$; in addition, under this measure, $X$ drifts to $+\infty$ (see Chapter 8 of [14] for details).

Observe that $X\left(\tau_{a, \gamma}^{+}\right)=S^{X}\left(\tau_{a, \gamma}^{+}\right)$for $\tau_{a, \gamma}^{+}<\infty$. Since

$$
a=U_{\gamma}\left(\tau_{a, \gamma}^{+}\right)=X\left(\tau_{a, \gamma}^{+}\right)-\gamma\left(S^{X}\left(\tau_{a, \gamma}^{+}\right)-u\right)
$$

for $\tau_{a, \gamma}^{+}<\infty$, we have

$$
X\left(\tau_{a, \gamma}^{+}\right) \mathbf{1}_{\left\{\tau_{a, \gamma}^{+}<\infty\right\}}=\frac{a-\gamma u}{1-\gamma} \mathbf{1}_{\left\{\tau_{a, \gamma}^{+}<\infty\right\}} .
$$

Since

$$
\frac{\mathrm{dP}_{u}^{\Phi(q)}}{\mathrm{dP}}=\exp \left(\Phi(q)\left(X\left(\tau_{a, \gamma}^{+}\right)-u\right)-q \tau_{a, \gamma}^{+}\right)
$$

on $\mathcal{F}_{\tau_{a, \gamma}^{+}}$, we then further obtain

$$
\begin{aligned}
\mathrm{E}_{u}\left[\exp \left(-q \tau_{a, \gamma}^{+}\right) \mathbf{1}_{\left\{\tau_{a, \gamma}^{+}<\tau_{0, \gamma}^{-}\right\}}\right] & =\mathrm{P}_{u}^{\Phi(q)}\left\{\tau_{a, \gamma}^{+}<\tau_{0, \gamma}^{-}\right\} \exp \left(-\Phi(q)\left(\frac{a-\gamma u}{1-\gamma}-u\right)\right) \\
& =\left(\frac{W_{\Phi(q)}(u)}{W_{\Phi(q)}(a)}\right)^{1 /(1-\gamma)} \exp \left(-\frac{\Phi(q)(a-u)}{1-\gamma}\right) \\
& =\left(\frac{\mathrm{e}^{-\Phi(q) u} W^{(q)}(u)}{\mathrm{e}^{-\Phi(q) a} W^{(q)}(a)}\right)^{1 /(1-\gamma)} \exp \left(-\frac{\Phi(q)(a-u)}{1-\gamma}\right)
\end{aligned}
$$

Therefore, the desired result follows readily.

Letting $q \rightarrow 0+$, we can obtain (3.1) for $q=0$.

\subsection{The survival probability}

Let

$$
\phi_{\gamma}(u)=\mathrm{P}_{u}\left\{\inf _{t \geq 0} U_{\gamma}(t) \geq 0\right\}
$$

denote the survival probability in the risk model with tax rate $\gamma$ and initial surplus $u$. Hence, $\phi_{0}(u)$ is the survival probability in the risk model without tax. For the compound Poisson risk model, Albrecher and Hipp [1] established a simple relation between the survival probability 
of a risk model with and without tax. We will now utilise Theorem 3.1 to generalise this result to spectrally negative Lévy risk processes.

Corollary 3.1. If $\gamma<1$ then

$$
\phi_{\gamma}(u)=\left(\phi_{0}(u)\right)^{1 /(1-\gamma)}
$$

Proof. From Theorem 3.1, we have

$$
\phi_{\gamma}(u)=\left(\psi^{\prime}(0+) W(u)\right)^{1 /(1-\gamma)},
$$

since $\lim _{a \rightarrow \infty} W(a)=\left(\psi^{\prime}(0+)\right)^{-1}$. The result follows from (2.2).

Note that $\phi_{\gamma}(u)>0$ if and only if $\phi_{0}(u)=\psi^{\prime}(0+)>0$, which is the case under the net profit condition $\mathrm{E}_{u}[X(1)-u]>0$.

\subsection{The discounted tax payments}

Let $\tau_{\gamma}:=\tau_{0, \gamma}^{-}$be the time of ruin of the risk process with tax and, furthermore, let

$$
T_{\gamma, \delta}=\gamma \int_{0}^{\tau_{\gamma}} \mathrm{e}^{-\delta t} \mathrm{~d} D(t)
$$

denote the present value of all tax payments until the time of ruin $\tau_{\gamma}$, where $D(t)=S^{X}(t)-X(0)$ and $\delta \geq 0$ can be interpreted as the force of interest.

Recall from [19] that

$$
V_{1}(u, u)=\frac{W^{(\delta)}(u)}{\left(W^{(\delta)}\right)^{\prime}(u)},
$$

where $V_{1}(u, u)$ is the expectation of the present value of all dividends paid until ruin when a horizontal barrier is at level $u$. Utilising a methodology from [19] for horizontal barrier models, we will now compute $v_{\gamma, \delta}(u)=\mathrm{E}_{u}\left[T_{\gamma, \delta}\right]$. Note that $v_{1, \delta}(u)=V_{1}(u, u)$ (so that the case in which $\gamma=1$ is settled).

Theorem 3.2. If $\gamma<1$ and $\delta>0$ then the expected discounted sum of tax payments until ruin is given by

$$
v_{\gamma, \delta}(u)=\frac{\gamma}{1-\gamma} \int_{u}^{\infty}\left(\frac{W^{(\delta)}(u)}{W^{(\delta)}(s)}\right)^{1 /(1-\gamma)} \mathrm{d} s .
$$

Proof. If $X(0)=u$ then

$$
S^{X}\left(\tau_{u+1 / n, \gamma}^{+}\right)-u=\frac{1 / n}{1-\gamma}
$$

Clearly, we have

$$
v_{\gamma, \delta}(u)=\mathrm{E}_{u}\left[T_{\gamma, \delta} ; \tau_{u+1 / n, \gamma}^{+}<\tau_{\gamma}\right]+\mathrm{E}_{u}\left[T_{\gamma, \delta} ; \tau_{u+1 / n, \gamma}^{+}>\tau_{\gamma}\right]
$$


For the second expression on the right-hand side, we have, using integration by parts,

$$
\begin{aligned}
\mathrm{E}_{u}\left[T_{\gamma, \delta} ; \tau_{u+1 / n, \gamma}^{+}>\tau_{\gamma}\right] \leq & \mathrm{E}_{u}\left[\int_{0}^{\tau_{u+1 / n, \gamma}^{+}} \mathrm{e}^{-\delta t} \gamma \mathrm{d}\left(S^{X}(t)-u\right) ; \tau_{u+1 / n, \gamma}^{+}>\tau_{\gamma}\right] \\
= & \mathrm{E}_{u}\left[\exp \left(-\delta \tau_{u+1 / n, \gamma}^{+}\right) \frac{\gamma(1 / n)}{1-\gamma} ; \tau_{u+1 / n, \gamma}^{+}>\tau_{\gamma}\right] \\
& +\mathrm{E}_{u}\left[\gamma \delta \int_{0}^{\tau_{u+1 / n, \gamma}^{+}} \mathrm{e}^{-\delta t}\left(S^{X}(t)-u\right) \mathrm{d} t ; \tau_{u+1 / n, \gamma}^{+}>\tau_{\gamma}\right] \\
\leq & \frac{2 \gamma / n}{1-\gamma}\left(1-\left(\frac{W(u)}{W(u+1 / n)}\right)^{1 /(1-\gamma)}\right) \\
= & o\left(\frac{1}{n}\right) .
\end{aligned}
$$

For the first expression on the right-hand side of (3.3), we can write

$$
\begin{aligned}
\mathrm{E}_{u}\left[T_{\gamma, \delta} ;\right. & \left.\tau_{u+1 / n, \gamma}^{+}<\tau_{\gamma}\right] \\
= & \mathrm{E}_{u}\left[\int_{0}^{\tau_{u+1 / n, \gamma}^{+}} \mathrm{e}^{-\delta t} \gamma \mathrm{d}\left(S^{X}(t)-u\right) ; \tau_{u+1 / n, \gamma}^{+}<\tau_{\gamma}\right] \\
& +\mathrm{E}_{u}\left[\int_{\tau_{u+1 / n, \gamma}^{+}}^{\tau_{\gamma}} \mathrm{e}^{-\delta t} \gamma \mathrm{d}\left(S^{X}(t)-u\right) ; \tau_{u+1 / n, \gamma}^{+}<\tau_{\gamma}\right] .
\end{aligned}
$$

Consider the two summands on the right-hand side separately. For (3.4), we apply the integration by parts formula to obtain

$$
\begin{aligned}
& \mathrm{E}_{u}\left[\int_{0}^{\tau_{u+1 / n, \gamma}^{+}} \mathrm{e}^{-\delta t} \gamma \mathrm{d}\left(S^{X}(t)-u\right) ; \tau_{u+1 / n, \gamma}^{+}<\tau_{\gamma}\right] \\
& =\mathrm{E}_{u}\left[\exp \left(-\delta \tau_{u+1 / n, \gamma}^{+}\right) \frac{\gamma / n}{1-\gamma} ; \tau_{u+1 / n, \gamma}^{+}<\tau_{\gamma}\right] \\
& \quad+\mathrm{E}_{u}\left[\gamma \delta \int_{0}^{\tau_{u+1 / n, \gamma}^{+}} \mathrm{e}^{-\delta t}\left(S^{X}(t)-u\right) \mathrm{d} t ; \tau_{u+1 / n, \gamma}^{+}<\tau_{\gamma}\right]
\end{aligned}
$$

where the second expression on the right-hand side can be bounded by

$$
\begin{aligned}
& \mathrm{E}_{u}\left[\gamma \delta \int_{0}^{\tau_{u+1 / n, \gamma}^{+}} \mathrm{e}^{-\delta t}\left(S^{X}(t)-u\right) \mathrm{d} t ; \tau_{u+1 / n, \gamma}^{+}<\tau_{\gamma}\right] \\
& \quad \leq \frac{\gamma / n}{1-\gamma}\left(\left(\frac{W(u)}{W(u+1 / n)}\right)^{1 /(1-\gamma)}-\left(\frac{W^{(\delta)}(u)}{W^{(\delta)}(u+1 / n)}\right)^{1 /(1-\gamma)}\right) \\
& \quad=o\left(\frac{1}{n}\right) .
\end{aligned}
$$

For (3.5), using the strong Markov property and the spatial homogeneity of the Lévy process $X$, we obtain

$$
\begin{aligned}
& \mathrm{E}_{u}\left[\int_{\tau_{u+1 / n, \gamma}^{+}}^{\tau_{\gamma}} \mathrm{e}^{-\delta t} \gamma \mathrm{d}\left(S^{X}(t)-u\right) ; \tau_{u+1 / n, \gamma}^{+}<\tau_{\gamma}\right] \\
& \quad=\mathrm{E}_{u}\left[\exp \left(-\delta \tau_{u+1 / n, \gamma}^{+}\right) ; \tau_{u+1 / n, \gamma}^{+}<\tau_{\gamma}\right] v_{\gamma, \delta}\left(u+\frac{1}{n}\right) .
\end{aligned}
$$


Consequently,

$$
v_{\gamma, \delta}(u)=\left(\frac{W^{(\delta)}(u)}{W^{(\delta)}(u+1 / n)}\right)^{1 /(1-\gamma)}\left(\frac{\gamma / n}{1-\gamma}+v_{\gamma, \delta}\left(u+\frac{1}{n}\right)\right)+o\left(\frac{1}{n}\right) .
$$

Standard algebraic manipulations to form the differential quotient for $v_{\gamma, \delta}$ then yield, for $n \rightarrow \infty$,

$$
\left(v_{\gamma, \delta}\right)^{\prime}(u)=\frac{\gamma}{1-\gamma}\left(\frac{\left(W^{(\delta)}\right)^{\prime}(u)}{\gamma W^{(\delta)}(u)} v_{\gamma, \delta}(u)-1\right) .
$$

This is the analogue of Equation (14) of [1]. Using the integrating factor technique for ordinary differential equations, we find that its solution is given by

$$
v_{\gamma, \delta}(u)=\left(C-\frac{\gamma}{1-\gamma} U_{2}(u)\right) \exp \left(\frac{U_{1}(u)}{1-\gamma}\right)
$$

for some constant $C$, where

$$
U_{1}(u)=\int_{0}^{u} \frac{\left(W^{(\delta)}\right)^{\prime}(s)}{W^{(\delta)}(s)} \mathrm{d} s, \quad U_{2}(u)=\int_{0}^{u} \exp \left(\frac{-U_{1}(s)}{1-\gamma}\right) \mathrm{d} s .
$$

We have $\left(W^{(\delta)}\right)^{\prime}(s) / W^{(\delta)}(s) \geq 0$ and

$$
\lim _{s \rightarrow \infty} \frac{\left(W^{(\delta)}\right)^{\prime}(s)}{W^{(\delta)}(s)}=\Phi(\delta) .
$$

The latter result can be found in [2] or [20]. Hence, $U_{1}$ is unbounded because $\Phi(\delta)>0$ for $\delta>0$. Also, since $\tau_{\gamma} \rightarrow \infty$ as $u \rightarrow \infty$ (for any $\gamma$ ), we have $\lim _{u \rightarrow \infty} v_{\gamma, \delta}(u)<\infty$. Thus,

$$
\lim _{u \rightarrow \infty} U_{2}(u)=\frac{1-\gamma}{\gamma} C,
$$

and then

$$
\begin{aligned}
v_{\gamma, \delta}(u)= & \frac{\gamma}{1-\gamma} \exp \left((1-\gamma)^{-1} \int_{0}^{u} \frac{\left(W^{(\delta)}\right)^{\prime}(s)}{W^{(\delta)}(s)} \mathrm{d} s\right) \\
& \times \int_{u}^{\infty} \exp \left(-(1-\gamma)^{-1} \int_{0}^{s} \frac{\left(W^{(\delta)}\right)^{\prime}(t)}{W^{(\delta)}(t)} \mathrm{d} t\right) \mathrm{d} s
\end{aligned}
$$

from which (3.2) follows.

Remark 3.1. If $X$ has a negative drift (i.e. $\left.\mathrm{E}_{u}[X(1)-u]<0\right)$ then (3.2) also holds for $\delta=0$.

Remark 3.2. Using (3.7), we can also write

$$
\begin{aligned}
v_{\gamma, \delta}(u)= & \frac{\gamma}{1-\gamma} \exp \left((1-\gamma)^{-1} \int_{0}^{u}\left(V_{1}(s, s)\right)^{-1} \mathrm{~d} s\right) \\
& \times \int_{u}^{\infty} \exp \left(-(1-\gamma)^{-1} \int_{0}^{s}\left(V_{1}(t, t)\right)^{-1} \mathrm{~d} t\right) \mathrm{d} s,
\end{aligned}
$$

recovering Theorem 2 of [1] in our more general Lévy setting. 
Remark 3.3. Using L'Hôpital's rule, we recover the following interesting relation:

$$
\lim _{u \rightarrow \infty} v_{\gamma, \delta}(u)=\gamma \lim _{u \rightarrow \infty} V_{1}(u, u)
$$

A direct probabilistic reasoning to obtain this identity goes as follows. In the absence of ruin the only difference for the calculation of $v_{\gamma, \delta}(u)$ and $V_{1}(u, u)$ is that, whenever tax (dividend) payments start and last until the next deviation from the running maximum, in the tax case only the proportion $\gamma$ of the income is paid, whereas in the horizontal barrier case all the income is paid. The only further difference is then that the surplus level at the next payment stream is different, but this latter difference does not matter if the distance to the ruin boundary does not matter, which in the limit $u \rightarrow \infty$ is the case. Hence, we immediately arrive at (3.8).

Remark 3.4. We can also understand (3.2) from an intuitive point of view. Whenever the taxed process $U_{\gamma}$ is in a running maximum, its increase scales with a factor $\gamma /(1-\gamma)$ for the increase of the associated tax payments. So the integral in (3.2) can be interpreted as summing up the (appropriately discounted) contributions given that a new running maximum is reached (cf. Theorem 3.1).

\subsection{Higher moments}

We will now investigate higher moments of $T_{\gamma, \delta}$. Let $v_{\gamma, \delta}^{(k)}(u)$ be the $k$ th moment of $T_{\gamma, \delta}$ when the initial surplus is equal to $u$. Recall from [17], and also from [15], that

$$
V_{k}(u, u)=k ! \prod_{i=1}^{k} \frac{W^{(i \delta)}(u)}{\left(W^{(i \delta)}\right)^{\prime}(u)}
$$

where $V_{k}(u, u)$ is the $k$ th moment of the present value of all dividends paid until ruin when the horizontal barrier is at level $u$. Note that $v_{1, \delta}^{(k)}(u)=V_{k}(u, u)$. So we need to address only the case in which $\gamma<1$.

Theorem 3.3. If $\gamma<1$ and $\delta>0$ then the kth moment of the present value of tax payments until ruin is related to the $(k-1)$ th moment by

$$
v_{\gamma, \delta}^{(k)}(u)=\frac{k \gamma}{1-\gamma} \int_{u}^{\infty} v_{\gamma, \delta}^{(k-1)}(s)\left(\frac{W^{(k \delta)}(u)}{W^{(k \delta)}(s)}\right)^{1 /(1-\gamma)} \mathrm{d} s .
$$

Proof. Proceeding as in the proof of Theorem 3.2 and the proof of Proposition 1 of [17], we have

$$
\begin{aligned}
v_{\gamma, \delta}^{(k)}(u)= & k v_{\gamma, \delta}^{(k-1)}\left(u+\frac{1}{n}\right) \frac{\gamma(1 / n)}{1-\gamma}\left(\frac{W^{(k \delta)}(u)}{W^{(k \delta)}(u+1 / n)}\right)^{1 /(1-\gamma)} \\
& +v_{\gamma, \delta}^{(k)}\left(u+\frac{1}{n}\right)\left(\frac{W^{(k \delta)}(u)}{W^{(k \delta)}(u+1 / n)}\right)^{1 /(1-\gamma)}+o\left(\frac{1}{n}\right) .
\end{aligned}
$$

Furthermore, we obtain

$$
\left(v_{\gamma, \delta}^{(k)}\right)^{\prime}(u)=\frac{\gamma}{1-\gamma}\left(\frac{\left(W^{(k \delta)}\right)^{\prime}(u)}{\gamma W^{(k \delta)}(u)} v_{\gamma, \delta}^{(k)}(u)-k v_{\gamma, \delta}^{(k-1)}(u)\right) .
$$


Solving this ordinary differential equation leads to

$$
\begin{aligned}
v_{\gamma, \delta}^{(k)}(u)= & \frac{k \gamma}{1-\gamma} \exp \left((1-\gamma)^{-1} \int_{0}^{u} \frac{\left(W^{(k \delta)}\right)^{\prime}(s)}{W^{(k \delta)}(s)} \mathrm{d} s\right) \\
& \times \int_{u}^{\infty} v_{\gamma, \delta}^{(k-1)}(s) \exp \left(-(1-\gamma)^{-1} \int_{0}^{s} \frac{\left(W^{(k \delta)}\right)^{\prime}(t)}{W^{(k \delta)}(t)} \mathrm{d} t\right) \mathrm{d} s .
\end{aligned}
$$

The statement follows from simple algebraic manipulations.

Remark 3.5. From (3.10) we obtain, by L'Hôpital's rule,

$$
\lim _{u \rightarrow \infty} v_{\gamma, \delta}^{(k)}(u)=k \gamma \lim _{u \rightarrow \infty} v_{\gamma, \delta}^{(k-1)}(u) \frac{W^{(k \delta)}(u)}{\left(W^{(k \delta)}\right)^{\prime}(u)} .
$$

With (3.9) we can hence generalise the asymptotic relation (3.8) to arbitrary moments of tax and dividend payments, respectively:

$$
\lim _{u \rightarrow \infty} v_{\gamma, \delta}^{(k)}(u)=\gamma^{k} \lim _{u \rightarrow \infty} V_{k}(u, u) .
$$

The alternative probabilistic argument from Remark 3.3 also carries over to explain (3.11).

\subsection{Examples}

3.5.1. Cramér-Lundberg process with exponential claims. If $X$ is a compound Poisson process with exponential jumps (with Poisson parameter $\lambda$ and exponential parameter $\alpha$ ) then the scale functions are given by

$$
W^{(\delta)}(x)=\frac{(\alpha+\rho) \mathrm{e}^{\rho x}(1-\eta(x))}{c(\rho-r)}
$$

(see, e.g. [15]), where

$$
\eta(x)=\frac{\alpha+r}{\alpha+\rho} \mathrm{e}^{(r-\rho) x},
$$

and $\rho$ and $r$ are respectively the positive and negative solutions of the equation

$$
c R^{2}+(c \alpha-\lambda-\delta) R-\alpha \delta=0 .
$$

Plugging this expression into (3.2), we eventually arrive at the explicit formula

$$
\begin{aligned}
v_{\gamma, \delta}(u)= & \frac{\gamma}{\rho}(1-\eta(u))^{1 /(1-\gamma)} \\
& \times{ }_{2} F_{1}\left(\frac{1}{1-\gamma}, \frac{\rho}{(\rho-r)(1-\gamma)}, \frac{\rho}{(\rho-r)(1-\gamma)}+1 ; \eta(u)\right),
\end{aligned}
$$

which was already derived in [1]. Here

$$
{ }_{2} F_{1}(a, b, c ; z)=\frac{\Gamma(c)}{\Gamma(b) \Gamma(c-b)} \int_{0}^{1} t^{b-1}(1-t)^{c-b-1}(1-z t)^{-a} \mathrm{~d} t,
$$

where $c>b>0$ denotes the Gauss hypergeometric series. 
3.5.2. Brownian motion with drift. Let $X(t)=m t+\sigma B(t)$ be a Brownian motion with drift (with $m \neq 0$ and $\sigma>0$ ). As in this case $\psi(\lambda)=m \lambda+\frac{1}{2} \sigma^{2} \lambda^{2}$ and $\Phi(\alpha)=-\omega+\theta_{\alpha}$, we can verify that

$$
W^{(\delta)}(x)=\frac{1}{\sigma^{2} \theta_{\delta}}\left(\exp \left(\left(-\omega+\theta_{\delta}\right) x\right)-\exp \left(-\left(\omega+\theta_{\delta}\right) x\right)\right),
$$

where $\theta_{\delta}=\sqrt{m^{2}+2 \delta \sigma^{2}} / \sigma^{2}$ and $\omega=m / \sigma^{2}$ (see also [2]). In particular, we have

$$
W(x)=\frac{1}{m}\left(1-\exp \left(-\frac{2 m}{\sigma^{2}} x\right)\right) .
$$

Thus,

$$
v_{1, \delta}(u)=V_{1}(u, u)=\frac{\sigma^{2}}{2 m}\left(\exp \left(\frac{2 m}{\sigma^{2}} u\right)-1\right),
$$

which recovers Equation (2.20) of [10].

Also, if $\gamma<1$ and if $\delta>0$, then we obtain

$$
\begin{aligned}
v_{\gamma, \delta}(u)= & \frac{\gamma}{1-\gamma}\left(\exp \left(\left(\theta_{\delta}-\omega\right) u\right)\left(1-\exp \left(-2 \theta_{\delta} u\right)\right)\right)^{1 /(1-\gamma)} \\
& \times \int_{u}^{\infty}\left(\exp \left(\left(\theta_{\delta}-\omega\right) s\right)\left(1-\exp \left(-2 \theta_{\delta} s\right)\right)\right)^{-1 /(1-\gamma)} \mathrm{d} s
\end{aligned}
$$

Since $\theta_{\delta}>\omega$ when $\sigma>0$ and $\delta>0$, the above expression can be rewritten as

$$
\begin{aligned}
v_{\gamma, \delta}(u)= & \frac{\gamma}{1-\gamma}\left(\frac{\left(1-\exp \left(-2 \theta_{\delta} u\right)\right)^{1 /(1-\gamma)}}{\theta_{\delta}-\omega}\right) \\
& \times{ }_{2} F_{1}\left((1-\gamma)^{-1}, \frac{\theta_{\delta}-\omega}{2 \theta_{\delta}}, \frac{3 \theta_{\delta}-\omega}{2 \theta_{\delta}} ; \exp \left(-2 \theta_{\delta} u\right)\right) .
\end{aligned}
$$

\section{Optimality of the tax barrier}

As tax payments stop at ruin, it is natural to ask whether the expected discounted tax payments over the lifetime of the process can be optimised when tax payments are started only after the surplus has reached a certain level $M$ (see [1] for a corresponding study in the Cramér-Lundberg framework). Due to the strong Markov property, we clearly have

$$
v_{\gamma, \delta, M}(u)=\frac{W^{(\delta)}(u)}{W^{(\delta)}(M)} v_{\gamma, \delta}(M)
$$

for $u<M$ and $v_{\gamma, \delta, M}(u)=v_{\gamma, \delta}(u)$ for $u \geq M$ (as then tax payments start immediately). Hence, the goal is to maximise (4.1) with respect to $M$.

Assumption 4.1. In what follows we assume that each scale function is three times differentiable and that its first derivative is a strictly convex function (so that $\left(W^{(\delta)}\right)^{\prime \prime}(u)$ changes its sign from negative to positive at most once).

Assumption 4.1 is, for instance, fulfilled if the Lévy measure has a completely monotone density (see [16] for the strict convexity of $\left(W^{(\delta)}\right)^{\prime}$ and [5] for infinite differentiability). Among particular examples fulfilling Assumption 4.1 are the gamma process and the inverse Gaussian process (for more examples, see [16]). 

if

Differentiating (4.1) with respect to $M$, we find that $M_{0}$ is a critical point of $M \mapsto v_{\gamma, \delta, M}(u)$

$$
v_{\gamma, \delta}\left(M_{0}\right)=V_{1}\left(M_{0}, M_{0}\right) \quad \text { or, equivalently, } \quad\left(v_{\gamma, \delta}\right)^{\prime}\left(M_{0}\right)=1,
$$

where (3.6) was used for the latter equivalence. To specify the nature of this critical point, we use the second derivative:

$$
\left.\frac{\partial^{2} v_{\gamma, \delta, M}(u)}{\partial M^{2}}\right|_{M=M_{0}}=\frac{\gamma}{1-\gamma} \frac{W^{(\delta)}(u)}{\left(W^{(\delta)}\left(M_{0}\right)\right)^{2}} v_{\gamma, \delta}\left(M_{0}\right)\left(W^{(\delta)}\right)^{\prime \prime}\left(M_{0}\right) .
$$

Clearly, since $\lim _{M \rightarrow \infty} v_{\gamma, \delta, M}(u)=0$ for any $u$, there is a point $M^{\star} \in[0, \infty)$ where the function $M \mapsto v_{\gamma, \delta, M}(u)$ reaches its global maximum.

Remark 4.1. Note that $M \mapsto v_{\gamma, \delta, M}(u)$ cannot have a local minimum in [0, $\left.\infty\right)$. Indeed, if there existed a local minimum then, by virtue of $\lim _{M \rightarrow \infty} v_{\gamma, \delta, M}(u)=0$, there would have to exist a local maximum for a larger value $M$. But in view of (4.3) and the strict convexity of $\left(W^{(\delta)}\right)^{\prime}$, this cannot occur.

Similarly, we deduce that after a potential saddlepoint there cannot be a local maximum.

Recall that

$$
V_{1}^{\prime}(s, s)=1-\frac{W^{(\delta)}(s)\left(W^{(\delta)}\right)^{\prime \prime}(s)}{\left(\left(W^{(\delta)}\right)^{\prime}(s)\right)^{2}}
$$

and from Remark 3.3 that

$$
\lim _{u \rightarrow \infty} v_{\gamma, \delta}(u)<\lim _{u \rightarrow \infty} V_{1}(u, u) .
$$

Remark 4.2. From the above, it follows that $M \mapsto v_{\gamma, \delta, M}(u)$ also cannot have a saddlepoint $M_{0}$ in $[0, \infty)$. Indeed, otherwise from $v_{\gamma, \delta}\left(M_{0}\right)=V_{1}\left(M_{0}, M_{0}\right)$ and $\left(W^{(\delta)}\right)^{\prime \prime}\left(M_{0}\right)=0$, we can observe that

$$
V_{1}^{\prime \prime}\left(M_{0}, M_{0}\right)=\frac{-W^{(\delta)}\left(M_{0}\right)\left(W^{(\delta)}\right)^{\prime \prime \prime}\left(M_{0}\right)}{\left(\left(W^{(\delta)}\right)^{\prime}\left(M_{0}\right)\right)^{2}}
$$

and $\left(v_{\gamma, \delta}\right)^{\prime \prime}\left(M_{0}\right)=0$. Hence, the function $s \mapsto v_{\gamma, \delta}(s)-V_{1}(s, s)$ reaches a local minimum value of 0 at this point $M_{0}\left(\right.$ as $\left.\left(W^{(\delta)}\right)^{\prime \prime \prime}\left(M_{0}\right)>0\right)$, implying that $v_{\gamma, \delta}$ is greater than $V_{1}$ in a neighbourhood of $M_{0}$, so that this saddlepoint would have to be followed by a maximum or another saddlepoint, which itself is excluded by the convexity of $\left(W^{(\delta)}\right)^{\prime}(u)$.

As a consequence, (4.2) has at most one positive solution $M_{0}$. If $V_{1}(0,0) \leq v_{\gamma, \delta}(0)$ then, due to (4.4), such a solution $M_{0}>0$ exists and is the point of global maximum, i.e. $M^{\star}=M_{0}$.

If $V_{1}(0,0)>v_{\gamma, \delta}(0)$ then $M^{\star}=0$ (i.e. tax payments start immediately), as a solution of (4.2), by (4.4), would have to be accompanied by a second one, which cannot be the case. Note that $M^{\star}$ is independent of the initial surplus $u$.

From the above discussion, we obtain the following final result which extends Theorem 3 of [1].

Theorem 4.1. Suppose that the scale functions of $X$ are three times differentiable and that their first derivatives are strictly convex functions. If $V_{1}(0,0)>v_{\gamma, \delta}(0)$ then the optimal height $M^{\star}$ is equal to 0 . If $V_{1}(0,0) \leq v_{\gamma, \delta}(0)$ then the optimal height $M^{\star}$ is the unique positive solution of (4.2). The maximum value is thus given by

$$
v_{\gamma, \delta, M^{\star}}(u)= \begin{cases}V_{1}\left(u, M^{\star}\right) & \text { if } u<M^{\star}, \\ v_{\gamma, \delta}(u) & \text { if } u \geq M^{\star} .\end{cases}
$$


Proof. If $u<M^{\star}$ then

$$
v_{\gamma, \delta, M^{\star}}(u)=\frac{W^{(\delta)}(u)}{W^{(\delta)}\left(M^{\star}\right)} v_{\gamma, \delta}\left(M^{\star}\right)=\frac{V_{1}\left(u, M^{\star}\right)}{V_{1}\left(M^{\star}, M^{\star}\right)} v_{\gamma, \delta}\left(M^{\star}\right)=V_{1}\left(u, M^{\star}\right) .
$$

Otherwise, we start to pay taxes right away and $v_{\gamma, \delta, M^{\star}}(u)=v_{\gamma, \delta}(u)$.

\section{References}

[1] Albrecher, H. ANd Hipp, C. (2007). Lundberg's risk process with tax. Blätter der DGVFM 28, 13-28.

[2] Avram, F., Palmowski, Z. and Pistorius, M. R. (2007). On the optimal dividend problem for a spectrally negative Lévy process. Ann. Appl. Prob. 17, 156-180.

[3] Bertoin, J. (1996). Lévy Processes. Cambridge University Press.

[4] Bertoin, J. (1997). Exponential decay and ergodicity of completely asymmetric Lévy processes in a finite interval. Ann. Appl. Prob. 7, 156-169.

[5] Chan, T. and Kyprianou, A. E. (2008). Smoothness of scale functions for spectrally negative Lévy processes. Submitted.

[6] Chiu, S. N. AND Yin, C. (2005). Passage times for a spectrally negative Lévy process with applications to risk theory. Bernoulli 11, 511-522.

[7] Doney, R. A. (2005). Some excursion calculations for spectrally one-sided Lévy processes. In Séminaire de Probabilités XXXVIII, (Lecture Notes Math. 1857), Springer, Berlin, pp. 5-15.

[8] Furrer, H. (1998). Risk processes perturbed by $\alpha$-stable Lévy motion. Scand. Actuarial J. 1, 59-74.

[9] Garrido, J. AND Morales, M. (2006). On the expected discounted penalty function for Lévy risk processes. N. Amer. Actuarial J. 10, 196-218.

[10] Gerber, H. U. and ShiU, E. S. W. (2004). Optimal dividends: analysis with Brownian motion. N. Amer. Actuarial J. 8, 1-20.

[11] Huzak, M., Perman, M., ŠIKić, H. and VondračEK, Z. (2004). Ruin probabilities and decompositions for general perturbed risk processes. Ann. Appl. Prob. 14, 1378-1397.

[12] Klüppelberg, C. And Kyprianou, A. E. (2006). On extreme ruinous behaviour of Lévy insurance risk processes. J. Appl. Prob. 43, 594-598.

[13] Klüppelberg, C., Kyprianou, A. E. And Maller, R. A. (2004). Ruin probabilities and overshoots for general Lévy insurance risk processes. Ann. Appl. Prob. 14, 1766-1801.

[14] Kyprianou, A. E. (2006). Introductory Lectures on Fluctuations of Lévy Processes with Applications. Springer, Berlin.

[15] Kyprianou, A. E. and Palmowski, Z. (2007). Distributional study of de Finetti's dividend problem for a general Lévy insurance risk process. J. Appl. Prob. 44, 428-443.

[16] Loeffen, R. (2008). On optimality of the barrier strategy in de Finetti's dividend problem for spectrally negative Lévy processes. To appear in Ann. Appl. Prob.

[17] Renaud, J.-F. and Zhou, X. (2007). Distribution of the present value of dividend payments in a Lévy risk model. J. Appl. Prob. 44, 420-427.

[18] Yang, H. And Zhang, L. (2001). Spectrally negative Lévy processes with applications in risk theory. Adv. Appl. Prob. 33, 281-291.

[19] Zhou, X. (2006). Discussion on: On optimal dividend strategies in the compound Poisson model, by H. Gerber and E. Shiu (N. Amer. Actuar. J. 10, 76-93). N. Amer. Actuarial J. 10, 79-84.

[20] ZHou, X. (2007). Exit problems for spectrally negative Lévy processes reflected at either the supremum or the infimum. J. Appl. Prob. 44, 1012-1030. 